\title{
Neurologic Compromise in COVID-19: A Literature Review
}

\author{
Leonardo Jardim Vaz de Mello ${ }^{\mathrm{a}, \mathrm{b}}$, h, Emylle Guimaraes Silva ${ }^{\mathrm{c}}$, Gabriel Oliveira Correa Rabelo ${ }^{\mathrm{c}}$, \\ Mariana Evaristo Leite ${ }^{\mathrm{c}}$, Nathalia Ramos Vieirac ${ }^{\mathrm{c}}$, Maryam Bahadori ${ }^{\mathrm{d}}$, Ali Seifie, \\ Daniel Agustin Godoye, f, g
}

\begin{abstract}
Coronavirus disease 2019 (COVID-19) disease caused by a new coronavirus, severe acute respiratory syndrome coronavirus 2 (SARS$\mathrm{CoV}-2$ ), has been associated with many neurological symptoms. The purpose of this article is to describe the neurological manifestations so far reported and their probable pathogenesis. We conducted a literature review on EMBASE, MEDLINE and SCIELO databases using the terms "COVID-19", "COVID", "neurological", "neurologic", "manifestations", "implications", "Guillain-Barre syndrome", "encephalopathy". A total of 33 articles including clinical series, retrospective studies, and case reports were selected and thoroughly reviewed to describe neurological manifestations of COVID-19. There are several neurological manifestations of SARS-CoV-2 infection with different clinical presentations, severity, and prevalence. The most critical ones, such as cerebrovascular disease, encephalopathy, and Guillain-Barre syndrome, were less common and usually associated with previous medical history, known risk factors for cerebrovascular disease or advanced age. The main hypotheses for the spread of the virus are through the hematogenous route or the cribriform plate of the ethmoid bone or a disseminated severe immune response by a cytokine storm. The presence of neurological disturbances associated with laboratory tests alterations is an important clue for the physicians to promptly recognize neurological manifestations of SARS-CoV-2.
\end{abstract}

Keywords: Coronavirus; SARS-CoV-2; COVID-19; Neurological

Manuscript submitted July 30, 2020, accepted August 6, 2020

Published online September 4, 2020

aDepartment of Medicine, School of Medicine, Federal University of Sao Joao del-Rei (UFSJ), Sao Joao del-Rei Campus, Sao Joao del-Rei, Brazil

${ }^{\text {b}}$ Departmento de Neurologia, Universidad Federal de Sao Joao del-Rei, Plaza Frei Orlando 170, Centro Sao Joao del-Rei, Minas Gerais, CEP: 36307-352, Brazil

'School of Medicine, Federal University of Sao Joao del-Rei (UFSJ), Sao Joao del-Rei Campus, Sao Joao del-Rei, Brazil

dUniversity of Texas Health San Antonio, San Antonio, TX, USA

eDepartment of Neurosurgery, University of Texas Health at San Antonio, San Antonio, TX, USA

${ }^{\mathrm{f}}$ Neurointensive Care Unit, Sanatorio Pasteur, Catamarca, Argentina Intensive Care Unit, Hospital Carlos Malbran, Catamarca, Argentina

${ }^{\mathrm{h} C o r r e s p o n d i n g ~ A u t h o r: ~ L e o n a r d o ~ J a r d i m ~ V a z ~ d e ~ M e l l o, ~ D e p a r t m e n t o ~ d e ~ N e u-~}$ rologia, Universidad Federal de Sao Joao del-Rei, Plaza Frei Orlando 170, Centro Sao Joao del-Rei, Minas Gerais, CEP: 36307-352, Brazil.

Email: ljvmello@ufsj.edu.br

doi: https://doi.org/10.14740/jnr619 manifestations; Guillain-Barre syndrome; Anosmia; Ageusia; Encephalitis

\section{Introduction}

Since the appearance of severe acute respiratory syndrome coronavirus 2 (SARS-CoV-2) in December 2019 until the last week of May 2020, the World Health Organization registered $5,206,614$ cases and 337,736 deaths. Up to writing date of this article, 216 countries have been affected by this pandemic, and accordingly the economic, financial, social, and mental havoc along with severe lockdown measures is of great concern. And the damage in the healthcare, mental, social, educational and economic areas, combined with severe lockdown measures, is a major concern. It has been demonstrated that the virus dissemination is through respiratory droplets, fomites and person-to-person contact. Transmission by stool removal has also been reported in healthcare workers, while taking care of infected patients [1-3].

Coronavirus disease 2019 (COVID-19) is a disease caused by a new coronavirus which can provoke several implications that vary from simple cold symptoms to the severe acute respiratory syndrome of which fever, cough and shortness of breath are the most common ones. Regarding neurological manifestations, anosmia and ageusia were the most common ones; however, cerebrovascular disease, headache, encephalopathy, Guillain-Barre syndrome (GBS) and myalgia have been reported as well. It is important to highlight these symptoms, so clinicians can consider SARS-CoV-2 as a probable diagnosis if they are the first symptoms to appear and suggest social isolation.

Mao et al have demonstrated that patients with severe COVID-19 are more likely to develop neurological symptoms such as acute cerebrovascular accidents, damage to skeletal muscle, and altered level of consciousness when compared to those with mild infection [4].

Initially, it was believed that SARS-CoV-2 manifested itself as a systemic flu syndrome affecting the respiratory tract. As the pandemic progressed, the presence of neurological symptoms of both the central nervous system (CNS) and the peripheral nervous system were noted. Such symptoms aroused the need to understand the pathophysiology of this virus in the nervous system and the mechanisms of its neuropathogenesis. The principal aim of this manuscript is to describe the neuro- 


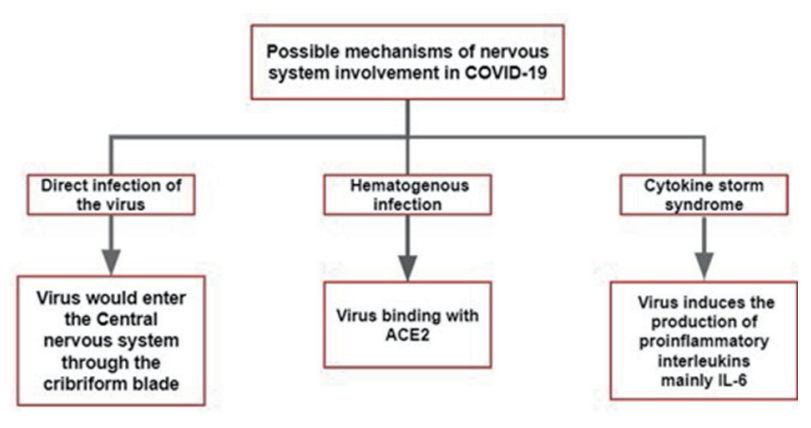

Figure 1. The main hypothesis of the pathophysiological mechanism of COVID-19 in the nervous system. COVID-19: coronavirus disease 2019; ACE: angiotensin-converting enzyme; IL: interleukin.

logical manifestations of COVID-19 which have been reported so far, the severe immune response triggered by it, and the potential neuropathogenesis.

\section{Literature Search}

We performed a literature search on EMBASE, MEDLINE and SCIELO databases using the terms "COVID-19", "COVID", "neurological", "neurologic", manifestations", "implications", "Guillain-Barre syndrome", "what about SARS-CoV-2".

\section{Literature Review}

\section{Pathophysiology}

Currently, there are several hypotheses for the pathophysiological mechanism in the nervous system which are listed as follows. The first is direct infection of the virus, a mechanism proposed to justify the anosmia and hyposmia of patients [4]. The virus could spread directly through the cribriform blade after penetrating the upper airway, which is also explained by the trans-synaptic spread [5]. The second is hematogenous infection. Several studies have described the role of angiotensin-converting enzyme 2 (ACE2), as a functional receptor for SARS-CoV-2. Paniz-Mondolfi et al showed the presence of the virus in neuronal endothelial cells $[6,7]$. The sluggish cerebral blood flow allows greater interaction between the virus and neuronal cells. Some pathophysiological mechanisms are still unknown, such as the presence of cerebral hemorrhagic complications in some patients with COVID-19 [6]. Third, cytokine storm syndrome (CSS) is another proposal for SARSCoV-2 injury to the nervous system. The neurotropism of the virus induces the production of interleukin-6 (IL-6) by glial cells. Thereafter, secondary to the action of neuroinflammatory cytokines, there is a chronic inflammatory and consequently a brain damage. There is also activation of the immune system detected for the presence of $\mathrm{CD}^{+}$, justifying the immune-mediated profile of the infection. Based on the aforementioned fact, it is evident that CSS is one of the many pathways used by SARS-CoV-2 to damage the brain indirectly $[3,8]$. Figure
1 summarizes the pathophysiological mechanism of nervous system involvement in COVID-19 infection.

\section{Neurological manifestations}

Table 1 summarizes the neurological manifestations of COVID-19 [4, 9-25].

\section{Discussion}

Li et al suggested that the acute respiratory failure in patients with COVID-19 may not only be caused by the direct damage to the lungs, but also due to the brain stem damage [26].

SARS-CoV-2 is $79 \%$ genetically identical to SARS-CoV and $50 \%$ to Middle East respiratory syndrome coronavirus (MERS-CoV) [27]. Both SARS-CoV and SARS-CoV-2 use ACE2 as the main functional receptor [28], while MERS-CoV uses dipeptidyl peptidase 4 (DPP4, also known as CD26). Beyond the respiratory symptoms, SARS-CoV and MERS-CoV can provoke many neurologic conditions [29,30]; this matter is emphasized by the presence of viral nucleic acid in cerebrospinal fluid (CSF) and in a brain autopsy [31]. Table 1 summarizes the most significant neurological manifestations. As a mechanism to reach the CNS, neurotropic viruses like coronaviruses use anterograde and retrograde transport with the assistance of kinesins and dynein motor proteins, through the sensory and motor nerve endings [32], particularly through the afferent nerve endings of the vagus nerve in lungs [26]. Furthermore, when SARS-CoV-2 causes a gastrointestinal tract infection, it can reach CNS by the enteric and sympathetic afferent nerve [33].

Based on the cytokine storm theory, Fotuhi et al proposed three different stages of neurological involvement in COVID-19. The first stage could be caused by low and controlled cytokine release limited to nasal and gustatory epithelial cells. Patients usually experience a temporary loss of smell and taste which is typically a benign course [34].

The second stage may be induced by a strong immune response expressed by elevated ferritin, C-reactive protein (CRP), and D-dimer blood levels. This inflammation state leads to hypercoagulopathy, vasculitis and direct immune-mediated damage to nerves and muscles. The related symptoms may be fatigue, hemiplegia, sensory loss, aphasia and ataxia. The third stage could be triggered by an even more severe cytokine storm that compromises the blood brain barrier which eases the infiltration of more inflammatory factors and viral particles into CNS. As a result, it leads to brain damage and injury that may cause delirium, encephalopathy, and/or seizures. In addition, the virus binds to the ACE2 receptor increasing peripheral vascular resistance causing hypertension which increases the risk of intracranial hemorrhage [34]. Figure 2 summarizes this physiopathological mechanism of neurological involvement.

\section{Anosmia and ageusia}

The neurological deficits are reported in uncomplicated and 

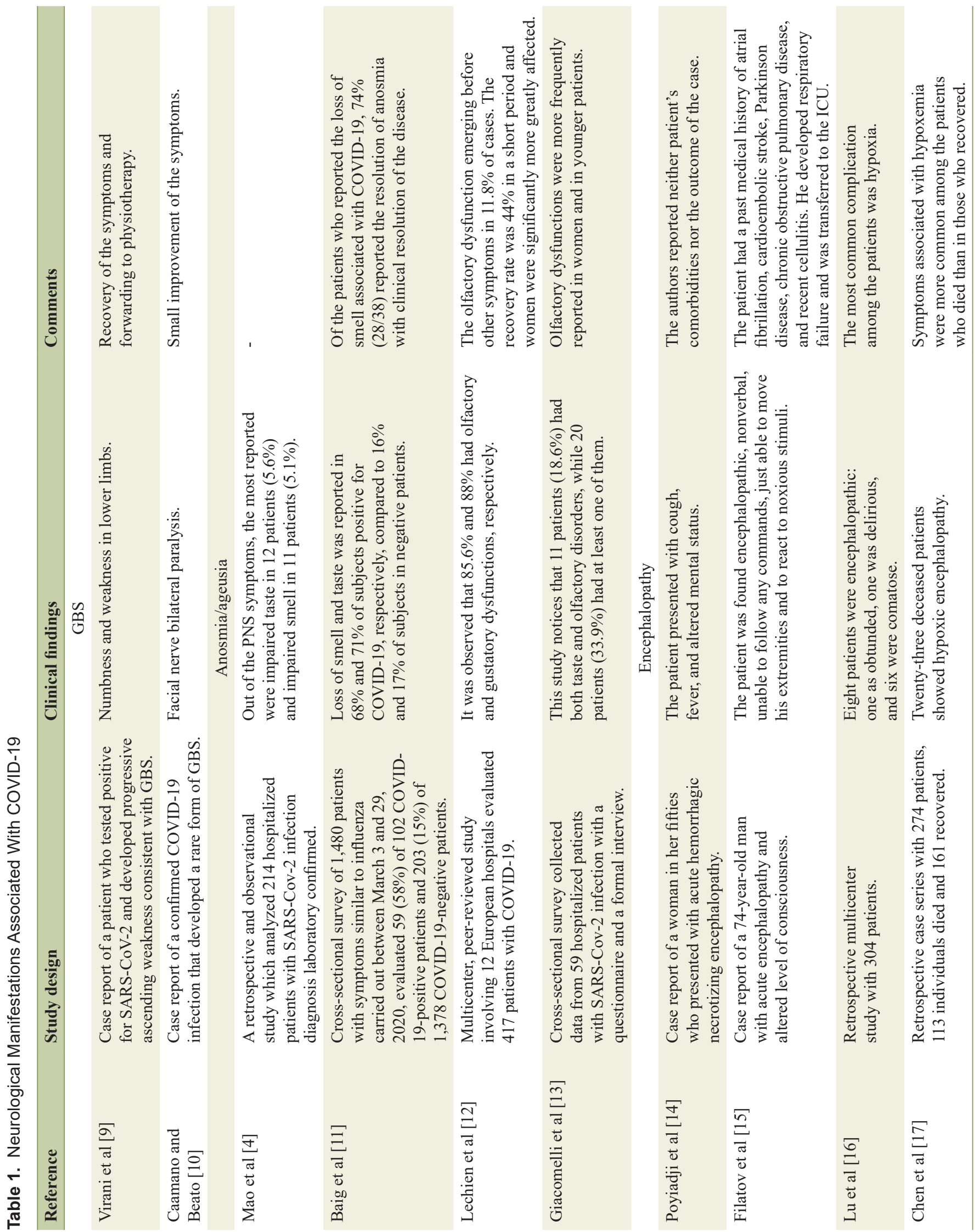


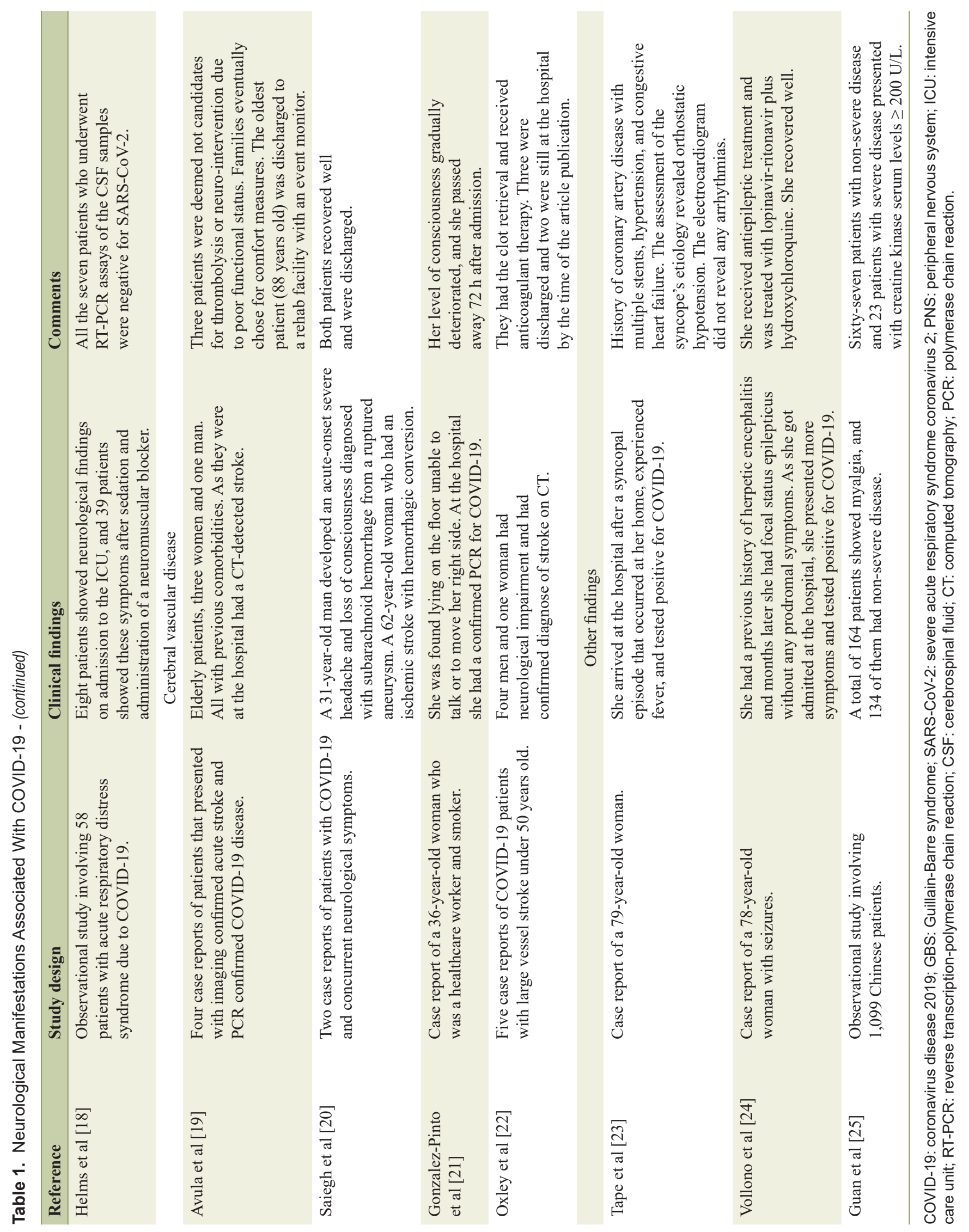




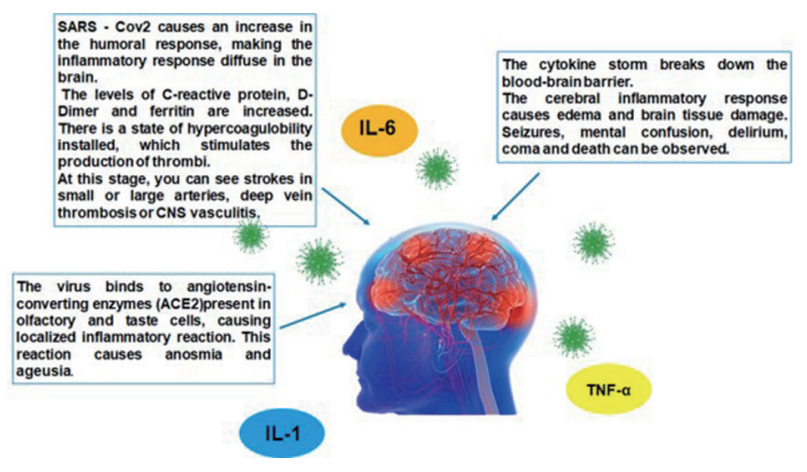

Figure 2. Illustrative scheme of brain cytokine storm effects. SARSCoV-2: severe acute respiratory syndrome coronavirus 2; ACE: angiotensin-converting enzyme; IL: interleukin; TNF: tumor necrosis factor; CNS: central nervous system.

complicated patients with COVID-19 worldwide. It is sufficient to establish that this deficit can be ongoing in patients with COVID-19 without being noticed. SARS-CoV-2 causing COVID-19 can take two routes to reach the brain. The hematogenous route seems to be the most probable way for SARSCoV-2 to reach the brain, but the other route to the CNS which is through the cribriform plate on the ethmoid bone, near the olfactory bulb, needs to be taken into account in the early-stage cases. Early occurrences of loss of smell, ataxia, and seizures should be evaluated for SARS-CoV-2 involvement in CNS [35]. The presence of neurological deficits associated with laboratory tests such as serum urea, creatinine, electrolytes, and blood gases $\left(\mathrm{PO}_{2}, \mathrm{PCO}_{2}\right)$ may be useful to determine primary or secondary involvement of the CNS in patients with COVID-19 [11].

Due to the high contagiousness and rapid spread of SARSCoV-2 and zero to mild symptoms in early stages of the disease, observation of patients with subclinical symptoms is crucial. Sudden anosmia and ageusia can help to immediately identify and isolate infected patients.

In a retrospective and observational study by Mao et al, 214 hospitalized patients with laboratory confirmed SARSCoV-2 infection were evaluated [4]. The data were collected from January 16, 2020 to February 19, 2020, at three care centers designated for COVID-19 (Main District, West Branch and Tumor Center) at Union Hospital of Huazhong University of Science and Technology in Wuhan, China. Out of the sample, 78 patients $(36.4 \%)$ were reported to have manifestations associated with the nervous system. These manifestations were divided into three subgroups: CNS, peripheral nervous system (PNS), and skeletal-related injury. In PNS subgroup, impaired taste, impaired sense of smell, impaired vision, and nerve pain were evaluated. Among the PNS symptoms, the most reported were impaired taste in 12 patients $(5.6 \%)$ and impaired smell in 11 patients $(5.1 \%)$ [4].

In a multicenter peer-reviewed study involving 12 European hospitals, Lechien et al evaluated 417 patients with COVID-19 and observed that $85.6 \%$ and $88 \%$ had olfactory and gustatory dysfunctions, respectively. The olfactory dysfunction occurred before other symptoms in $11.8 \%$ of cases. The recovery rate was $44 \%$ in a short period and women were significantly more greatly affected [12].
In a cross-sectional survey, Giacomelli et al collected data from 59 hospitalized patients with SARS-CoV-2 infection with a questionnaire and a formal interview. This study noticed that 11 patients $(18.6 \%)$ had both taste and olfactory disorders, while 20 patients $(33.9 \%)$ had at least one of these symptoms. Furthermore, $20.3 \%$ and $91 \%$ had, respectively, anosmia and ageusia before hospitalization. Olfactory dysfunctions were more frequently reported in women as well as younger patients. All patients reported the persistence of olfactory and gustatory disorders at the time of the interview [13]. Reinforcing these findings, in another study, approximately 150 doctors from the Daegu Medical Association prospectively collected data from anosmia and ageusia cases by interviewing 3,191 patients over the phone in Daegu, Korea. In the early stage of COVID-19, anosmia or acute ageusia was observed in $15.3 \%$ of the 3,191 patients and, in the group of asymptomatic to mild disease patients $(2,342)$, these symptoms were observed in $15.7 \%$. Its prevalence was significantly more common among women and younger individuals $(\mathrm{P}=0.01$ and $\mathrm{P}<0.001$, respectively). Most patients with anosmia or ageusia recovered within 3 weeks. The average recovery time was 7 days for both symptoms [36].

Kaye et al surveyed 237 COVID-19 patients in the USA and found that anosmia was observed in 73\% which was the initial symptom in $26.6 \%$. Some improvement was observed in $27 \%$ of patients, with an average improvement time of 7.2 days ( $85 \%$ of this group showed improvement in 10 days) [37].

In a cross-sectional survey carried out between March 3 and 29 of 2020 by Yan et al, loss of smell and taste was reported in $68 \%$ and $71 \%$ of subjects positive for COVID-19, respectively, compared to $16 \%$ and $17 \%$ of subjects in COVID-19-negative patients. The impairment of smell and taste was independently and strongly associated with the positivity of COVID-19 (anosmia: adjusted odds ratio (aOR) 10.9, 95\% confidence interval (CI): 5.08 - 23.5; ageusia: aOR 10, 95\% CI: 4.74 - 22.1), while sore throat was associated with COVID-19 negativity (aOR $0.23,95 \% \mathrm{CI}: 0.11-0.50)$. Of the patients who reported loss of smell associated with COVID-19, 74\% (28/38) reported resolution of anosmia with clinical resolution of the disease [35].

Thus, it is observed that anosmia and ageusia appear as common symptoms in COVID-19, ranging from $5.1 \%$ to $85.6 \%$ for olfactory disorders and $5.6 \%$ to $88 \%$ for gustatory disorders. Sudden anosmia or ageusia should be recognized by the international scientific community as important symptoms of COVID-19 infection, especially in the initial stage of the disease. During the COVID-19 pandemic, doctors should be suspicious of SARS-CoV-2 infection if they encounter patients with these symptoms to avoid delayed diagnosis and break the chain of infections. These symptoms are more prevalent in women and younger people. The clinical resolution of most patients occurs on average in 7 days, with the majority recovering within 3 weeks. It was also found that these sensory impairments were more prevalent in patients who developed less severe disease manifestations [4].

\section{Cerebral vascular disease}

Although some authors have claimed that hospital visits for 
cerebrovascular events have been reduced during COVID-19 pandemic, several cases of cerebrovascular diseases have been reported as comorbidities of COVID-19. However, such diseases at $5.7 \%$ of total neurological implications of COVID-19 are considered as less common neurological complications [1]. There are few reports of ischemic stroke as initials manifestation of COVID-19; the authors considered only patients who had the ictus before the hospitalization and had positive polymerase chain reaction (PCR) test for SARS-CoV-2 [19, 20]. Most patients were elderly, although some studies have reported cases in patients of less than 50 years old $[21,22]$. In addition, the majority of them had previous comorbidities or cardiovascular risk factors. In general, most of these patients had a poor clinical outcome.

There are some hypotheses about the possible causes of stroke due to COVID-19 infection, but none of them have been proven yet. The most accepted one is that the coagulopathy is induced by the systemic inflammatory response and this theory is reinforced by finding high level of D-dimer and CRP levels in most cases. In addition, antiphospholipid antibodies (aPLs) were detected in three patients [38]. Another theory would be the depletion of ACE2 and endothelial dysfunction. The ACE2 is expressed in lung, endothelial cells, glial cells and neurons. It acts by protecting the brain and other organs from ischemia as it disables the renin-angiotensin-aldosterone system by cleaving angiotensin-II to angiotensin or to angiotensin-I that is also converted in angiotensin. Angiotensin induces vasodilatation and has anti-inflammatory effects. As SARS-CoV-2 enters the cell, it depletes ACE2 through receptor endocytosis while ACE-1 that activates the renin-angiotensin-aldosterone system is unopposed [39]. The treatment measures were mechanical thrombectomy and thrombolysis [19, 22]. Platelet anti-aggregant and statins were also used.

Unfortunately, some patients were not candidates for thrombolysis or neuro-intervention [19]. There are some new drugs being tested such as recombinant human soluble ACE2 and angiotensin [39].

Other cerebral vascular diseases which were reported as less common COVID-19 complications were aneurysmal subarachnoid hemorrhage, cerebral venous sinus thrombosis, and cerebral hemorrhage.

\section{Encephalopathy}

The first case of acute hemorrhagic necrotizing encephalopathy was reported by Poyiadji et al [14]. This case was a COVID-19-positive woman who presented with cough, fever and altered mental status. Analysis of the CSF was negative to bacterial growth, herpes simplex viruses 1 and 2, varicella zoster virus and West Nile virus. The presence of SARS-CoV-2 was not tested in the CSF sample. On magnetic resonance imaging (MRI) study the hemorrhagic rim enhancing lesions were found in the medial temporal lobes, bilateral thalami, and subinsular lesions.

In addition, non-contrast head computed tomography (CT) images demonstrated the presence of a symmetric hypo-attenuation in the bilateral medial thalami where CT angiogram and CT venogram were normal [14].
Filatov et al described a case of a 74-year-old man who presents with headache, altered mental status, cough, and fever 1 week after arriving to the USA from Netherlands. He had a past medical history of atrial fibrillation, cardioembolic stroke, Parkinson disease, chronic obstructive pulmonary disease, and cellulitis. The patient was encephalopathic, nonverbal, and unable to follow any commands without nuchal rigidity. He was able to move all his extremities and answered to noxious stimuli. Head CT was normal, but the electroencephalography showed bilateral slowing and focal slowing in the left temporal region with sharply countered waves. Analysis of the CSF was normal. The patient was transferred to the intensive care unit (ICU) due to the progression of the disease [15].

In a retrospective multicenter study by Lu et al, 304 individuals with coronavirus disease were evaluated among which eight patients were encephalopathic (six were comatose, one was delirious, and one was obtunded) [16]. In Chen et al study, among 113 patients who died of COVID-19, 23 (20\%) individuals developed hypoxic encephalopathy as a complication of COVID-19 [17].

Helms et al developed an observational study with 58 French patients with COVID-19. In the admission to ICU, eight patients showed neurological symptoms, and after the withdrawal of the sedation and neuromuscular blocker this number increased to 39 patients. Seven patients had a past medical history of previous neurologic disorders as transient ischemic attack, partial epilepsy, and mild cognitive impairment. Due to unexplained encephalopathic features, 13 patients underwent MRI of the brain, of which eight had enhancement in leptomeningeal spaces. All the 11 patients who underwent perfusion imaging had bilateral frontotemporal hypoperfusion. Electroencephalography was performed in eight patients in whom nonspecific changes were found and one patient had also diffuse bifrontal slowing, suggestive of encephalopathy. The CSF analysis performed on seven patients showed neither cells nor SARS-CoV in the reverse transcription-polymerase chain reaction (RT-PCR) assays [18].

There is still no clear data about the physiological pathways that lead to these manifestations. It was noticed that patients who had previously neurological conditions and showed acute respiratory symptoms were more likely to have encephalopathy [15]. Hypoxia was the most common risk factor among the patients evaluated by Lu et al [16]. Other conditions that may be related to encephalopathy in COVID-19 patients are the cytokines storm, the critical illness-related encephalopathy, the withdrawal of medications, and even a possible direct effect caused by SARS-CoV-2 infection [18].

\section{GBS}

The GBS is caused by an accentuated immune response that generates cross-reactivity to gangliosides peripherical components causing demyelination. It is believed that IL-6 plays an important role in the malfunction of several organs which can be fatal to patients with COVID-19. Probably the same immune mechanisms are involved with neurological complications. It was observed that people who developed severe respiratory syndrome were the ones who also had severe neu- 
rological complications [40].

Patients diagnosed with GBS commonly complain about facial inclination, diplopia, dysarthria, dysphagia, ophthalmoplegia, pupillary disorders, paresthesia, numbness, tachycardia, bradycardia, facial flushing, paroxysmal hypertension, orthostatic hypotension, anhidrosis and/or diaphoresis and urinary retention. The main respiratory symptom is dyspnea on exertion and eventually most patients need support from an ICU to deal with respiratory failure [41].

It is believed that the histopathological mechanism of GBS is related to Campylobacter jejuni infection. This microorganism has capsule antigens similar to antigens on nerve cells resulting in the cross immune response to the ganglioside GM1 in myelin, causing damage to the peripheral nervous system. The histopathological findings are lymphocytic infiltration of the roots of the spine and peripheral nerves, followed by macrophage-mediated multifocal myelin removal. This process causes a change in the propagation of nerve impulses, with eventual absence or profound delay in conduction, causing flaccid paralysis. Recovery is usually associated with remyelination [41].

Although GBS is mostly associated with campylobacteriosis, viral infections such as cytomegalovirus, Epstein-Barr virus, human immunodeficiency virus and Zika virus have also been reported to be associated with this syndrome. It is also possible to find an intrinsic relationship with COVID-19. There is a case report, reinforcing the mentioned possibility, of a patient that was in an areflexic paralytic state with ascendant symmetrical weakness, fever, and cough. The mentioned neurological symptoms usually happen 3 days to 6 weeks after the infection. In this case, COVID-19 was probably the trigger illness [9].

Most reports of GBS related to COVID-19 involved the elderly and presented as an acute areflexic quadriparesis. Severe respiratory failure was also observed due to lobar pneumonia and interstitial pneumonitis. They had motor axonal and motor sensory axonal polyneuropathy as well [42].

In addition, in a case report, facial diplegia, facial symmetrical weakness, and ocular weakness along with albuminocytological dissociation in CSF, were observed in a patient 10 days after SARS-CoV-2 confirmed infection. The final conjecture is that SARS-CoV-2 infection may have triggered this atypical clinical variant of GBS in the patient [10].

\section{Other findings}

Although fever and respiratory symptoms have been commonly used to identify suspects of COVID-19, Tape et al have demonstrated a different case [23], a 79-year-old woman, who arrived at the hospital after a syncope episode at her home. Initially, she was afebrile with a normal chest X-ray and there was no suspicion of COVID-19. Then she experienced fever and tested positive for COVID-19. The patient had a history of coronary artery disease with multiple stents, hypertension, and congestive heart failure. An assessment of the syncope's etiology revealed orthostatic hypotension with blood pressure of $116 / 62 \mathrm{~mm} \mathrm{Hg}$ in the supine position and $85 / 50 \mathrm{~mm} \mathrm{Hg}$ when standing. The electrocardiogram did not reveal any ar- rhythmias. The chest CT showed bilateral peripheral groundglass opacity. She was discharged home after the improvement in symptoms and resolution of the dizziness. At the time of discharge, she continued with low-grade intermittent fever of 38 to $38.2{ }^{\circ} \mathrm{C}$.

Later, she got worse at home and was readmitted to another hospital with respiratory arrest. Thus, a normal chest X-ray and atypical signs of infection such as syncope cannot disregard COVID-19. This unique case highlights the increased diversity of COVID-19 presentations, errors in initial diagnosis, and delays in implementing the suitable precautions [23].

Seizures were also reported as neurologic manifestations of COVID-19 [3, 24]. There is a case report of a 78-year-old woman with a previous history of a herpetic encephalopathy 2 years ago with no seizure episodes since then. In this case the focal status epilepticus was the only manifestation of the SARS-CoV-2 infection [24]. In 2004, a case of a 32-year-old pregnant woman who had COVID infection was reported. She had tonic-colonic convulsion and the PCR exam of the CSF was positive for SARS-CoV [43]. As both viruses are very similar taxonomically, it is important to relate this report.

In a study among 1,099 patients evaluated by Guan et al, $164(14.9 \%)$ presented with myalgia [25]. In another study conducted by Chen et al, 25 (19\%) of the 113 deceased patients also had this symptom [17]. Skeletal muscle injury was reported by $10.7 \%$ of the patients in the study of Mao et al. The authors noticed that patients with muscular symptoms showed higher creatine kinase and lactate dehydrogenase levels compared with patients without these symptoms. The muscle injury may occur due to infection-mediated harmful immune response, elevated proinflammatory cytokines or the interaction between SARS-CoV-2 and the ACE2 in the skeletal muscle [4].

Another symptom was headache that may happen by a variety of causes. One of them is the direct invasion of the virus to the nervous system causing damage to nerves resulting in pain. Another theory is hypoxia caused by alveolar gas exchange disturbance so the anaerobic metabolism in mitochondria of cerebral cells increases. Therefore, an acid accumulation occurs that can provoke cerebral vasodilatation, cerebral cell swelling, interstitial edema, obstruction of the brain blood flow and consequently headache [44].

\section{Conclusions}

Several neurological manifestations of SARS-CoV-2 infection have different presentations, severity and prevalence. The most critical ones, such as cerebrovascular disease, encephalopathy, GBS, were less common and usually associated with previous medical history illness, vascular disease risk factors, or advanced age. The mechanisms of neurological implications are not well determined yet. The main hypotheses are: 1) The spread of the virus through hematogenous route; 2) The cribriform lamina of the ethmoid bone; and 3) Disseminated severe immune response by cytokine storm. The presence of neurological disturbances associated with lab exams' alterations is an important clue for doctors to identify SARS-CoV-2 
infection, promote social isolation, and offer adequate support. As COVID-19 is a new emergent highly infective disease, there is still lack of studies with stronger levels of evidence and larger study populations. Therefore, the neurological manifestation studies are limited to retrospective studies and case reports.

\section{Acknowledgments}

None to declare.

\section{Financial Disclosure}

None to declare.

\section{Conflict of Interest}

None to declare.

\section{Author Contributions}

LJVM contributed to literature search and writing the manuscript; EGS, GOCR, MEL and NRV contributed to literature search; MB edited the paper; AS was involved in scientific editing of the paper; DAG contributed to manuscript design and editing the paper.

\section{Data Availability}

Any inquiries regarding supporting data availability of this study should be directed to the corresponding author.

\section{References}

1. WHO Coronavirus Disease (COVID-19) Dashboard. Data last updated: May 24, 2020. https:/covid19.who. int/.

2. WHO. Key messages and actions for COVID-19 prevention and control in schools. March 2020.

3. Ahmed MU, Hanif M, Ali MJ, Haider MA, Kherani D, Memon GM, Karim AH, et al. Neurological manifestations of COVID-19 (SARS-CoV-2): a review. Front Neurol. 2020;11:518.

4. Mao L, Jin H, Wang M, Hu Y, Chen S, He Q, Chang J, et al. Neurologic manifestations of hospitalized patients with coronavirus disease 2019 in Wuhan, China. JAMA Neurol. 2020.

5. Zubair AS, McAlpine LS, Gardin T, Farhadian S, Kuruvilla DE, Spudich S. Neuropathogenesis and neurologic manifestations of the coronaviruses in the age of coronavirus disease 2019: A Review. JAMA Neurol. 2020.

6. Turner AJ, Hiscox JA, Hooper NM. ACE2: from vaso- peptidase to SARS virus receptor. Trends Pharmacol Sci. 2004;25(6):291-294.

7. Paniz-Mondolfi A, Bryce C, Grimes Z, Gordon RE, Reidy J, Lednicky J, Sordillo EM, et al. Central nervous system involvement by severe acute respiratory syndrome coronavirus-2 (SARS-CoV-2). J Med Virol. 2020;92(7):699702.

8. Whittaker A, Anson M, Harky A. Neurological Manifestations of COVID-19: A systematic review and current update. Acta Neurol Scand. 2020;142(1):14-22.

9. Virani A, Rabold E, Hanson T, Haag A, Elrufay R, Cheema T, Balaan M, et al. Guillain-Barre Syndrome associated with SARS-CoV-2 infection. IDCases. 2020:e0771.

10. Juliao Caamano DS, Alonso Beato R. Facial diplegia, a possible atypical variant of Guillain-Barre Syndrome as a rare neurological complication of SARS-CoV-2. J Clin Neurosci. 2020;77:230-232.

11. Baig AM. Neurological manifestations in COVID-19 caused by SARS-CoV-2. CNS Neurosci Ther. 2020;26(5):499-501.

12. Lechien JR, Chiesa-Estomba CM, De Siati DR, Horoi M, Le Bon SD, Rodriguez A, Dequanter D, et al. Olfactory and gustatory dysfunctions as a clinical presentation of mild-to-moderate forms of the coronavirus disease (COVID-19): a multicenter European study. Eur Arch Otorhinolaryngol. 2020;277(8):2251-2261.

13. Giacomelli A, Pezzati L, Conti F, Bernacchia D, Siano M, Oreni L, Rusconi S, et al. Self-reported olfactory and taste disorders in patients with severe acute respiratory coronavirus 2 infection: a cross-sectional study. Clin Infect Dis. 2020;71(15):889-890.

14. Poyiadji N, Shahin G, Noujaim D, Stone M, Patel S, Griffith B. COVID-19-associated acute hemorrhagic necrotizing encephalopathy: imaging features. Radiology. 2020;296(2):E119-E120.

15. Filatov A, Sharma P, Hindi F, Espinosa PS. Neurological complications of coronavirus disease (COVID-19): Encephalopathy. Cureus. 2020;12(3):e7352.

16. Lu L, Xiong W, Liu D, Liu J, Yang D, Li N, Mu J, et al. New onset acute symptomatic seizure and risk factors in coronavirus disease 2019: A retrospective multicenter study. Epilepsia. 2020;61(6):e49-e53.

17. Chen T, Wu D, Chen H, Yan W, Yang D, Chen G, Ma $\mathrm{K}$, et al. Clinical characteristics of 113 deceased patients with coronavirus disease 2019: retrospective study. BMJ. 2020;368:m1091.

18. Helms J, Kremer S, Merdji H, Clere-Jehl R, Schenck M, Kummerlen C, Collange O, et al. Neurologic Features in Severe SARS-CoV-2 Infection. N Engl J Med. 2020;382(23):2268-2270.

19. Avula A, Nalleballe K, Narula N, Sapozhnikov S, Dandu V, Toom S, Glaser A, et al. COVID-19 presenting as stroke. Brain Behav Immun. 2020;87:115-119.

20. Al Saiegh F, Ghosh R, Leibold A, Avery MB, Schmidt RF, Theofanis T, Mouchtouris N, et al. Status of SARS-CoV-2 in cerebrospinal fluid of patients with COVID-19 and stroke. J Neurol Neurosurg Psychiatry. 2020;91(8):846848.

21. Gonzalez-Pinto T, Luna-Rodriguez A, Moreno-Estebanez 
A, Agirre-Beitia G, Rodriguez-Antiguedad A, Ruiz-Lopez M. Emergency room neurology in times of COVID-19: malignant ischaemic stroke and SARS-CoV-2 infection. Eur J Neurol. 2020.

22. Oxley TJ, Mocco J, Majidi S, Kellner CP, Shoirah H, Singh IP, De Leacy RA, et al. Large-vessel stroke as a presenting feature of COVID-19 in the young. N Engl J Med. 2020;382(20):e60.

23. Tape C, Byrd KM, Aung S, Lonks JR, Flanigan TP, Rybak NR. COVID-19 in a patient presenting with syncope and a normal chest X-ray. R I Med J (2013). 2020;103(3):5051.

24. Vollono C, Rollob E, Romozzib M, et al. Focal status epilepticus as unique clinical feature of COVID-19: A case report. Seizure: European Journal of Epileps. 2020. https://doi.org/10.1016/j.seizure.2020.04.009.

25. Guan WJ, Ni ZY, Hu Y, Liang WH, Ou CQ, He JX, Liu L, et al. Clinical characteristics of coronavirus disease 2019 in China. N Engl J Med. 2020;382(18):1708-1720.

26. Li YC, Bai WZ, Hashikawa T. The neuroinvasive potential of SARS-CoV2 may play a role in the respiratory failure of COVID-19 patients. J Med Virol. 2020;92(6):552555.

27. Lu R, Zhao X, Li J, Niu P, Yang B, Wu H, Wang W, et al. Genomic characterisation and epidemiology of 2019 novel coronavirus: implications for virus origins and receptor binding. Lancet. 2020;395(10224):565-574.

28. Ge XY, Li JL, Yang XL, Chmura AA, Zhu G, Epstein $\mathrm{JH}$, Mazet JK, et al. Isolation and characterization of a bat SARS-like coronavirus that uses the ACE2 receptor. Nature. 2013;503(7477):535-538.

29. Tsai LK, Hsieh ST, Chang YC. Neurological manifestations in severe acute respiratory syndrome. Acta Neurol Taiwan. 2005;14(3):113-119.

30. Saad M, Omrani AS, Baig K, Bahloul A, Elzein F, Matin MA, Selim MA, et al. Clinical aspects and outcomes of 70 patients with Middle East respiratory syndrome coronavirus infection: a single-center experience in Saudi Arabia. Int J Infect Dis. 2014;29:301-306.

31. Schoeman D, Fielding BC. Coronavirus envelope pro- tein: current knowledge. Virol J. 2019;16(1):69.

32. Swanson PA, 2nd, McGavern DB. Viral diseases of the central nervous system. Curr Opin Virol. 2015;11:44-54.

33. Wong SH, Lui RN, Sung JJ. Covid-19 and the digestive system. J Gastroenterol Hepatol. 2020;35(5):744-748.

34. Fotuhi M, Mian A, Meysami S, Raji CA. Neurobiology of COVID-19. J Alzheimers Dis. 2020;76(1):3-19.

35. Yan CH, Faraji F, Prajapati DP, Boone CE, DeConde AS. Association of chemosensory dysfunction and COVID-19 in patients presenting with influenza-like symptoms. Int Forum Allergy Rhinol. 2020;10(7):806-813.

36. Lee Y, Min P, Lee S, Kim SW. Prevalence and duration of acute loss of smell or taste in COVID-19 patients. J Korean Med Sci. 2020;35(18):e174.

37. Kaye R, Chang CWD, Kazahaya K, Brereton J, Denneny JC, 3rd. COVID-19 anosmia reporting tool: initial findings. Otolaryngol Head Neck Surg. 2020;163(1):132134.

38. Zhang Y, Xiao M, Zhang S, Xia P, Cao W, Jiang W, Chen $\mathrm{H}$, et al. Coagulopathy and antiphospholipid antibodies in patients with COVID-19. N Engl J Med. 2020;382(17):e38.

39. Hess DC, Eldahshan W, Rutkowski E. COVID-19-related stroke. Transl Stroke Res. 2020;11(3):322-325.

40. Scheidl E, Canseco DD, Hadji-Naumov A, Bereznai B. Guillain-Barre syndrome during SARS-CoV-2 pandemic: A case report and review of recent literature. J Peripher Nerv Syst. 2020;25(2):204-207.

41. Andary MT, Oleszek JL, Maurelus K, White-McCrimmon R. Guillain-Barre syndrome. Medscape. 2020.

42. Sedaghat Z, Karimi N. Guillain Barre syndrome associated with COVID-19 infection: A case report. J Clin Neurosci. 2020;76:233-235.

43. Lau KK, Yu WC, Chu CM, Lau ST, Sheng B, Yuen KY. Possible central nervous system infection by SARS coronavirus. Emerg Infect Dis. 2004;10(2):342-344.

44. Wu Y, Xu X, Chen Z, Duan J, Hashimoto K, Yang L, Liu $\mathrm{C}$, et al. Nervous system involvement after infection with COVID-19 and other coronaviruses. Brain Behav Immun. 2020;87:18-22. 